\title{
Gaseous Detectors of Ultraviolet and Visible Photons
}

\author{
V. Peskov \\ Fermi National Accelerator Laboratory \\ P.O. Box 500, Batavia, Illinois 60510 \\ A. Borovik-Romanov \\ Institute for Physical Problems \\ Russia \\ T. Volynshikova \\ Institute of Metallurgy and Alloys \\ Russia
}

June 1994

Presented at the Symposium on Radiation Measurements and Applications, The University of Michigan, Ann Arbor, Michigan, May 16-19, 1994 


\section{Disclaimer}

This report was prepared as an account of work sponsored by an agency of the United States Government. Neither the United States Government nor any agency thereof, nor any of their employees, makes any warranty, express or implied, or assumes any legal liability or responsibility for the accuracy, completeness, or usefulness of any information, apparatus, product, or process disclosed, or represents that its use would not infringe privately owned rights. Reference herein to any specific commercial product, process, or service by trade name, trademark, manufacturer, or otherwise, does not necessarily constitute or imply its endorsement, recommendation, or favoring by the United States Government or any agency thereof. The views and opinions of authors expressed herein do not necessarily state or reflect those of the United States Government or any agency thereof. 


\title{
Gaseous detectors of ultraviolet and visible photons
}

\author{
V. Peskov \\ Fermi National Accelerator Laboratory, USA \\ A. Borovik-Romanov \\ Institute for Physical Problems, Russia \\ T. Volynshikova \\ Institute of Metallurgy and Alloys, Russia \\ Presented at Symposium on Radiation Measurements and Applications. May 16-19, 1994. \\ The University of Michigan, Ann Arbor, Michigan

\begin{abstract}
We describe simple methods of manufacturing in a laboratory gaseous detectors of visible photons with $\mathrm{GaAs}(\mathrm{Cs})$ and $\mathrm{SbCs}$ photocathodes and Ti getters. Covered by CsI protective layers they are robust enough to be stable under ordinary experimental conditions. First attempts to use these detectors for crystal scintillator and fiber readout are presented.
\end{abstract}




\section{Introduction}

Last years many efforts have been made by different groups to develop gaseous detectors of UV and visible photons with solid photocathodes (see for example [1,2] and references therein). The main advantages of such types of detectors are: much lower than gas threshold of spectral sensitivity, and a very good time resolution ( $<1 \mathrm{~ns}$ ). The first wire chambers with $\mathrm{CuI}$ photocathodes were built and used for plasma diagnostics [3]. Later other photocathodes were tested, such as TMPD and CsI [4]. The CsI photocathode has advantages compared to other ultraviolet photocathodes that it is air stable and has high quantum efficiency $(\mathrm{QE})$ for wavelength $\lambda<220 \mathrm{~nm}$. In a gas atmosphere the $\mathrm{QE}$ of solid photocathodes increases with the field near the cathode and this leads to a parallelplate design [5]. An important contribution was made by the authors of ref.[6-9] who have demonstrated that photocathodes with sensitivity up to visible light $(\lambda \sim 500 \mathrm{~nm})$ can be manufactured inside the gaseous detectors. Unfortunately stable operation of the photocathodes in gas atmosphere were achieved only at ultra clean conditions $[6-8,10]$ and this makes it difficult to manufacture this kind of detectors in a laboratory .

The main aim of this work was to develop a simple technology allowing the manufacture of gaseous detectors of visible photons in ordinarily experimental conditions such as: initial vacuum $\sim 10^{-6}$, outgassing at temperature $100-200^{\circ} \mathrm{C}$, not sealed detector, etc. We describe our latest achievements in this direction and discuss possible applications of the gaseous detectors of visible photons for crystal scintillators and fiber readout.

\section{Large area detectors}

It was observed earlier [11] that a rather stable operation of the SbCs and $\mathrm{GaAs}(\mathrm{Cs})$ photocathodes can be achieved in ordinary experimental conditions ( initial vacuum $10^{-6}$, heating only to $100 \mathrm{C}$ during the outgassing) with a getter inside the detector and at very low light flux ( equivalent charge flux to the cathode $<\mathrm{fA} / \mathrm{cm}^{2}$ ). In our previous work [11] a Cs layer, evaporated on the glass surface, was used as a getter. 
We consider this observation as a key issue in searching for a simple production technology of the gaseous detectors of visible photons. In this work we concentrated on improving this technique. Several other types of getter and different procedure of manufacturing were tested. The best results were achieved with a Ti getter. The schematic drawing of our detector with the getter inside is presented in fig.1. It is a stainless steel chamber inside of which is installed Sb or GaAs reflective photocathodes. The anode was a mesh placed a few mm away. A flange with a window is attached by using a $\mathrm{Cu}$ gasket. The getter was a $\mathrm{Ti}$ wire inside an open ended glass tube. The SbCs and $\mathrm{GaAs}(\mathrm{Cs})$ photcathodes were manufactured inside the detector by the method described in ref. [9]. Before the Sb or GaAs photocathode were coated by a Cs layer, the chamber was pumped during a few days to a vacuum better than $10^{-6}$ and heated to 100 $200^{\circ} \mathrm{C}$. After this preparation the getter was activated. The Ti wire is heated by current to dark red color and this gives a thin metallic coating inside the tube which serves as a getter. The advantage of this type of a getter is that, if necessary, it can be used many times. The detectors QE and stability achieved with the Ti getter are presented in fig.2 and 3. Compared to our previous results reported in ref.[9] we got very similar value for the $\mathrm{QE}$, but better long term stability.

We studied also the aging properties of our detectors. Typically the $\mathrm{QE}$ dropped by a factor of 2 at total collected charge of $50 \mu \mathrm{C} / \mathrm{cm}^{2}$. It is better than reported by other authors $[8,10]$ and can be attributed to the presence of the getter, adsorbing the outgasing and dissociation products.

Another way to reach stable operation is to use a protective layer on the photocathode surface [11]. Some layers, for example CsI, are transparent for the photoelectrons extracted from the photocathode covered by this layer [ 9]. In the first test [9] the photocathode covered with a CsI protective layer was exposed to air when mounted on the detector. Exposure to air caused cracks and the gas leaks through the cracks cause slow degradation of the photocathode. In our previous work [9] in order to avoid this effect we introduced also TMAE which, due to the surface tension, closed the holes and cracks and also served as a some sort of getter for oxygen.

In the current work we used a similar technology of covering the photocathode by a protective CsI layer, but without exposure to air. Our set up was the same as described in ref. [9], but modified in such a way that some mechanical manipulations were possible inside the bell jar after the evaporation had been finished-see fig.4. Two procedures were tested. In the first one, the detector chamber with a Sb photocathode and a mesh anode were installed inside the bell jar. If necessary the detector could be rotated and covered by a flange inside the bell jar by means of a special mechanical device. 
Immediately after the photocathode manufacturing (by evaporating Cs on the Sb plate) and upon coating it by a CsI protective layer the chamber was covered with flange and the gas was introduced from outside. The rubber O-ring was used in this design. The outside pressure kept the chamber sealed. Then the chamber was extracted from the bell jar, bolted, evacuated to better vacuum, and the getter was activated. All these procedures typically took $15-30 \mathrm{~min}$. Then the ultra clean $\mathrm{CH}_{4}(99.999 \%)$ was introduced into the detector chamber and the $\mathrm{QE}$ measured.

In the second procedure, after the photocathode was evaporated and covered by a protective layer, the clean $\mathrm{Ar}$ (99.9995\%) was introduced in to the bell jar. The bell jar was modified in such a way that a small glove box, flushed also with Ar, could be connected to it through the special vacuum line with a hatch. At pressure $1 \mathrm{~atm}$ in the bell jar the hatch can be opened and this allows manipulations inside the jar. While being flushed by the gas the chamber with photocathode was bolted and taken away. $\mathrm{A} \mathrm{Cu}$ gasket was used in this set up. Then the chamber was pumped, the getter activated and the clean gas introduced. The second method gave much better results probably because the $\mathrm{Cu}$ gasket was used. The $\mathrm{QE}$ of the SbCs photocathodes with 10 and $20 \mathrm{~nm}$ thick protective layer of the CsI are presented in fig.2. One can see that it is higher than original value for the SbCs in the short wavelength $(\lambda<210 \mathrm{~nm})$ and lower in the long wavelength. However the $\mathrm{QE}$ achieved at $\lambda>210 \mathrm{~nm}$ are considerably higher than in our previous work [9], when photocathodes with CsI protective layers were exposed to air during installation in to the test chamber. It is interesting to note also that the shape of the QE qualitatively confirms calculation of low energy electron transport through the CSI made in work [12]. The $\mathrm{S}$ bCs photocathodes with the CsI protective layer provide better long term stability than without (see fig.3)

Gas gains up to $10^{5}$ were achieved with the protective layers probably due to the lower $\mathrm{QE}$ in the long wavelength region. A linear response in a dynamic range of $10^{4}$ was observed.

We also observed that covering $C$ s based photocathodes with a 20-50 nm thick layer of CsI improved the aging properties by 2-3 orders of magnitude (the QE drops on a factor 2 at a collected charge density of $\sim 1 \mathrm{mC} / \mathrm{cm}^{2}$ ). Further investigations are needed to demonstrate the long term efficiency of such technology. But even the aging properties that already have been achieved are sufficient for some applications such as crystal readout.

We should note that very similar results were obtained with $\mathrm{GaAs}(\mathrm{Cs})$ photocathodes-see fig.5. 


\section{Small detectors}

We have made and successfully tested several small prototypes of such detectors with SbCs reflective and semitransparent photocathodes (see fig.6). The detectors were made from glass (inner diameter 2, 3,5 and $10 \mathrm{~mm}$ ) with $\mathrm{SbCs}$ photocathodes manufactured inside. The technology of covering the $\mathrm{Sb}$ cathodes inside the glass tubes by a Cs layer was as following. Cs was evaporated inside other glass volume B, connected to the detector volume A(see fig.6). By heating the walls of the tube $B$ Cs was moved to the detector volume $\mathrm{A}$, where it interacts with $\mathrm{Sb}$. By heating the detector $\mathrm{A}$ while keeping the tube $B$ at room temperature the excess $C s$ was moved then again to the volume $\mathrm{B}$. Then the tube $\mathrm{B}$ was disconnected and clean $\mathrm{CH}_{4}$ was introduced to the detector. Higher $\mathrm{QE}$ were achieved with reflective photocathodes and for this reason we basically concentrated on manufacturing this type of detectors. In ref.[11] we reported about the beginning of a long term stability test. The results that we have achieved so far are presented in fig. 3. We observed some degradation in time which was stronger in the case of small detectors. That probably can be explained by bigger surface to volume ratio

\section{Possible applications}

\subsection{Crystal Scintillators Readout}

Studies done by the "Crystal clear" collaboration identify recently two new promising candidates for the $\mathrm{CMS}$ detectors: $\mathrm{CeF}_{3}$ and $\mathrm{Pb}_{2} \mathrm{WO}_{4}$ [13]. $\mathrm{CeF}_{3}$ has high light yield, but is expensive. $\mathrm{Pb}_{2} \mathrm{WO}_{4}$ is twice as cheap, but emits 10 times less light. The gaseous detectors with $\mathrm{SbCs}$ and $\mathrm{GaAs} / \mathrm{Cs}$ photocathodes appear to be ideal for the readout of the $\mathrm{Pb}_{2} \mathrm{WO}_{4}$ scintillator. They can work at sufficiently high gains and in this way compensate the relatively low intensity of the scintillation light. We made some preliminary tests with a small $\left(\sim 1 \mathrm{~cm}^{3}\right)$ crystal of $\mathrm{Pb}_{2} \mathrm{WO}_{4}$ irradiated by ${ }^{106} \mathrm{Ru},{ }^{137} \mathrm{Cs}$ and $22 \mathrm{Na}$ sources. The small size of the crystal does not allow us to collimate well the sources and get good spectrum, so the results should be considered only as qualitative. Nevertheless we clearly observed the shape typical for the scintillation light produced by $\mathrm{Ru}$ and $\mathrm{Cs}$ and $\mathrm{Na}$ - see for example fig.7. Energy resolution $\sim 80 \%$ was obtained with $0.5 \mathrm{MeV}$ photons.

\subsection{Scintillating Fiber Readout}


We also made an attempt to detect the scintillation light from the fibers emitting the light at $440 \mathrm{~nm}$ by means of our small detectors- see fig. 6 As we mentioned before detectors with highly efficient photocathodes can work only at relatively low gas gain of $\sim 10^{3}$. For this reason we were able to record only the "tail" of the pulse-height spectra -see fig.8. Efficiency 1.5-2\% was achieved with respect to the triggered photomultiplier. Since the sensitivity of the gaseous detectors with SbCs photocathodes are considerably smaller than for PM or solid state detectors, their applicability is limited to the cases where a lot of light is emitted. Examples of such devices are lead/fiber pre-shower detectors or shower -max detectors. Of course the stability problem of the small detectors should be solved first. Since the QE degradation is less in the case of the large volume detector the best solution in future will be to use sealed gas microstrip detectors with a semitransparent photocathode.

\section{Conclusions}

We demonstrated that gaseous detectors with solid photocathodes can be manufactured in an ordinarily laboratory conditions. The protective CsI layers allow one to achieve satisfactory stability of air reactive photocathodes such as SbCs and GaAs without ultraclean conditions. Probably after some further development gas detectors with solid photocathodes could find applications in calorimetry, large area Cherenkov detectors and plasma diagnostics.

\section{Acknowledgments}

The authors thank M. Atac, A. Bross, G. Charpak and A. Ronjin for the discussion and encouragement throughout this work

\section{References:}

[1] G. Charpak et al., Nucl. Instr. and Meth.. A327 (1992) 445

[2] G. Charpak et al., Preprint CERN-PPE/92-220, 1992

[3] V. Peskov, Doct. of Science Thesis, Inst. for Phys. Problems, Moscow , 1981

[4] V. Peskov et al., Nucl. Instr. and Methods A269 (1988)149

J. Seguinot et al Nucl. Instr. and Methods A297 (1990) 133

[5] G. Charpak et al., Proceedings of the Sympos. on Particle Ident. at High Lum. Hadron Colliders, (eds. T. Gourlay and J. Morfin, Fermilab, Batavia, IL, 1989) p. 295 
[6] V. Peskov, Journ. of Appl. Spectr., 48 (1988) 316

[7] J.S. Edmends et al., Nucl. Instr. and Meth., A237, (1988) 145

[8] D.R. Winn IEEE Trans. Nucl. Sci. 36(1989), 128

[9] A. Borovik-Romanov et al., Preprint Fermilab-Conf- 93/250, 1993

[10] G. Charpak et al., Preprint CERN-EP/82-169,1982

S. Majewski et al., Preprint CERN-EP/83-89, 1983

[11] V. Peskov et al., Preprint Fermilab-Conf.-93/351, 1993

[12] A. Akkerman et al., Preprint Weizmann Inst. of Science WIS-94/12/Feb-PH

[13] P. Lecoq, "New results on crystal calorimeters", report at 4-th International Conference on Calorimetry in High Energy Physics, September 1993, Isola de Elba, Italy 


\section{Figure captions:}

Fig. 1. Schematic view of a large area gas detectors (inner diameter) with SbCs or GaAs (Cs) photocathodes and a Ti getter inside for the detecting the scintillating light from the crystals.

Fig.2. The QE of the SbCs photocathodes vs. the wavelength under different conditions: room temperature $(20 \mathrm{C}), 60 \mathrm{C}$ and without and with CsI protective layers. One can see that heating to $60 \mathrm{C}$ helped to increase the $\mathrm{QE}$. Similar effect was observed earlier in the case of the CsI photocathode and probably can be used in practice.

Fig.3. QE vs. time for the SbCs photocathode and for the different size of detectors ( numbers on the fig. correspond to the diameters). Lower curve corresponds to the SbCs photocathode, covered by a CsI protective layer. To make the drawing compact the $\mathrm{QE}$ for the SbCs+CsI photocathode is multiplied by 10 . All measurements were done in $\mathrm{CH}_{4}$ (20 Torr) at a gain of $\sim 10^{5}$.

Fig. 4. The set up used for covering the SbCs and GaAs photocathodes by a CsI protective layer without exposure it to air.

Fig. 5. The QE of the GaAs photocathodes vs. wavelength with and without CsI protective layers

Fig. 6. Schematic drawing of the sealed compact gaseous detectors with CsI photocathodes for readout of individual scintillating fibers.

Fig. 7. Scintillation light from $\mathrm{Pb}_{2} \mathrm{WO}_{4}$ crystal recorded by the gaseous detector with SbCs photocathode. Gas gain $\sim 10^{4}$. a) Source- ${ }^{106} \mathrm{Ru}$. b) ${ }^{137} \mathrm{Cs}$.

Fig.8 Scintillation light from fibers recorded by a gaseous detector with inner diameter $10 \mathrm{~mm}$. 


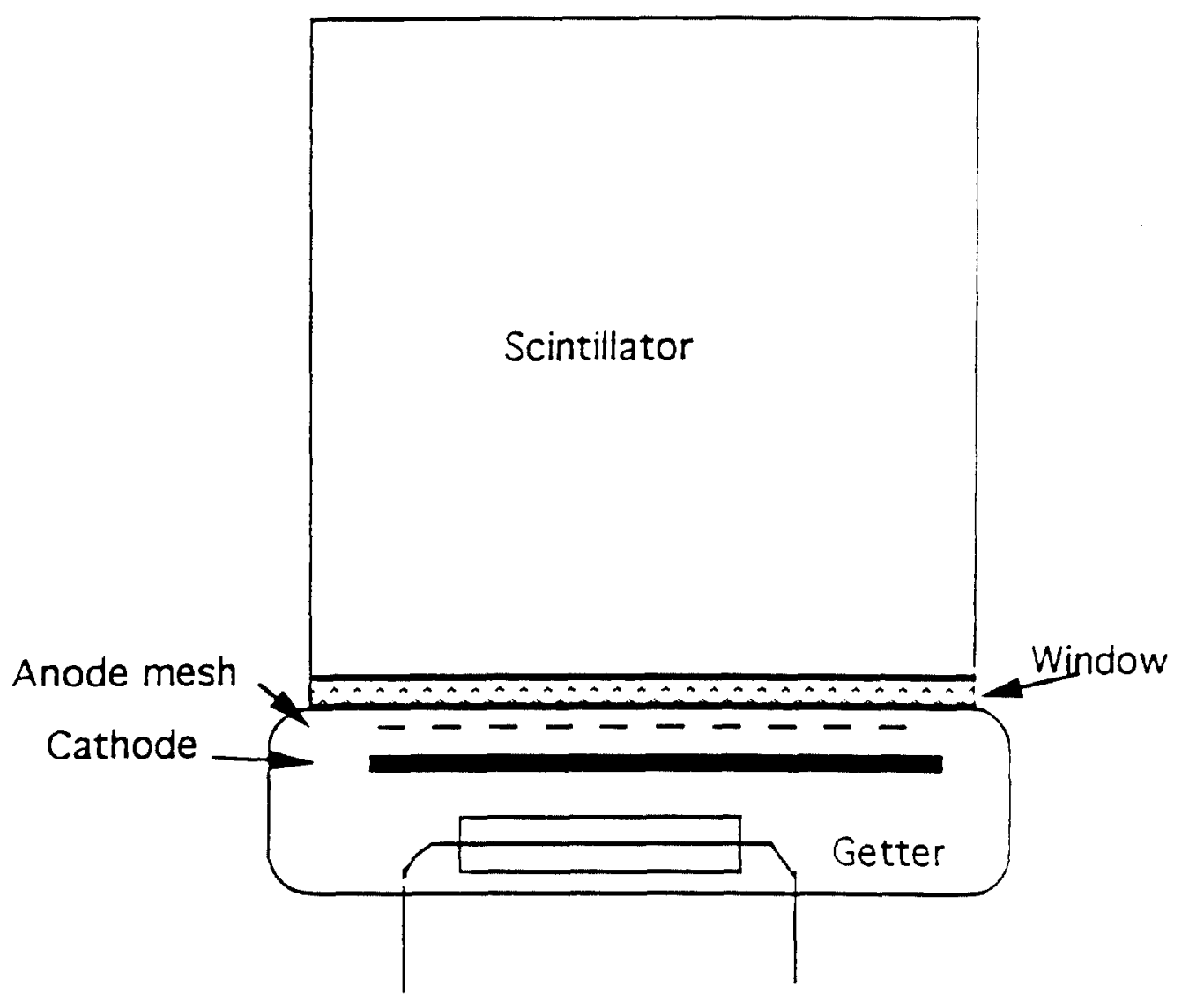

Fig. 1 


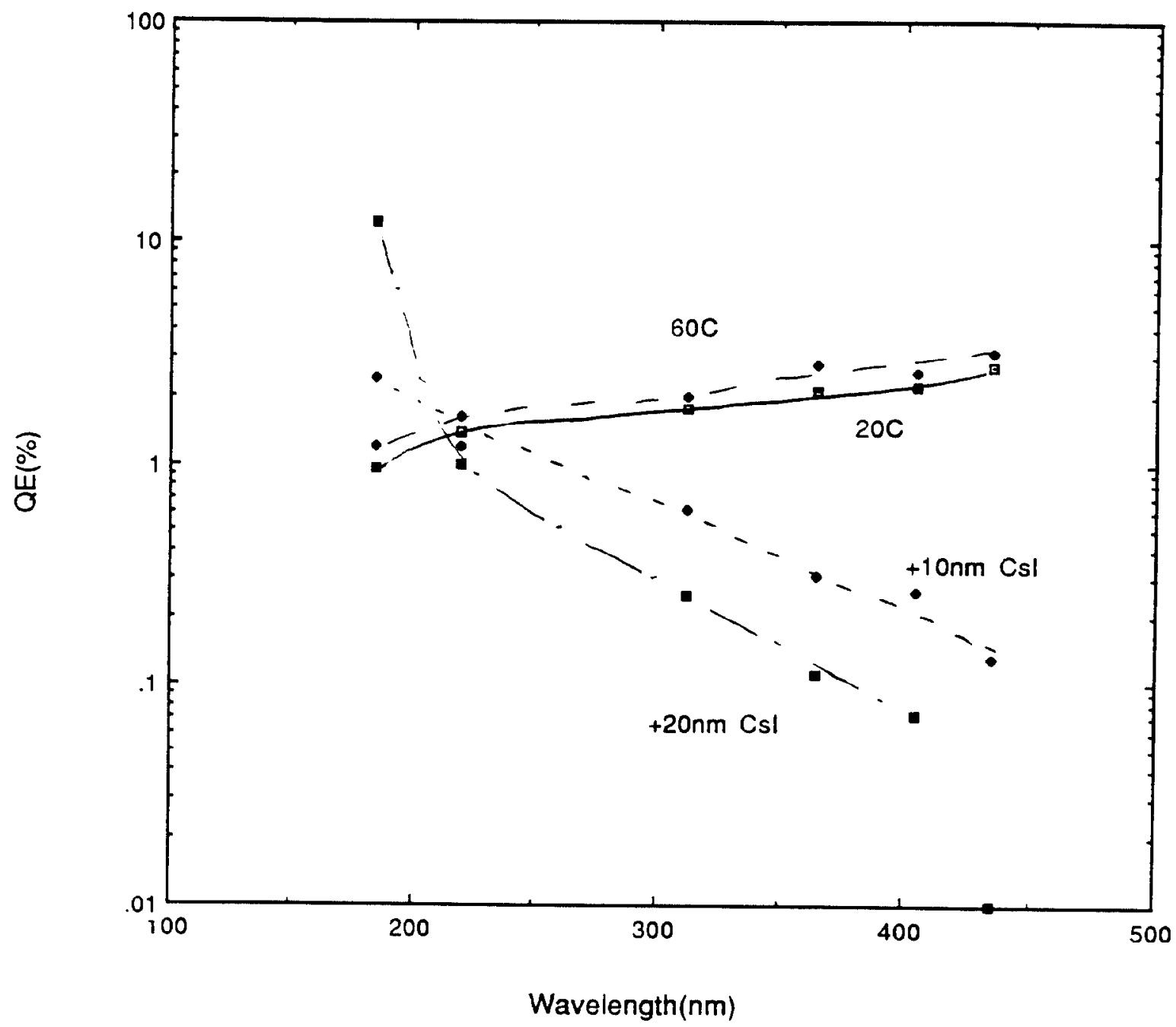

Fig. 2 


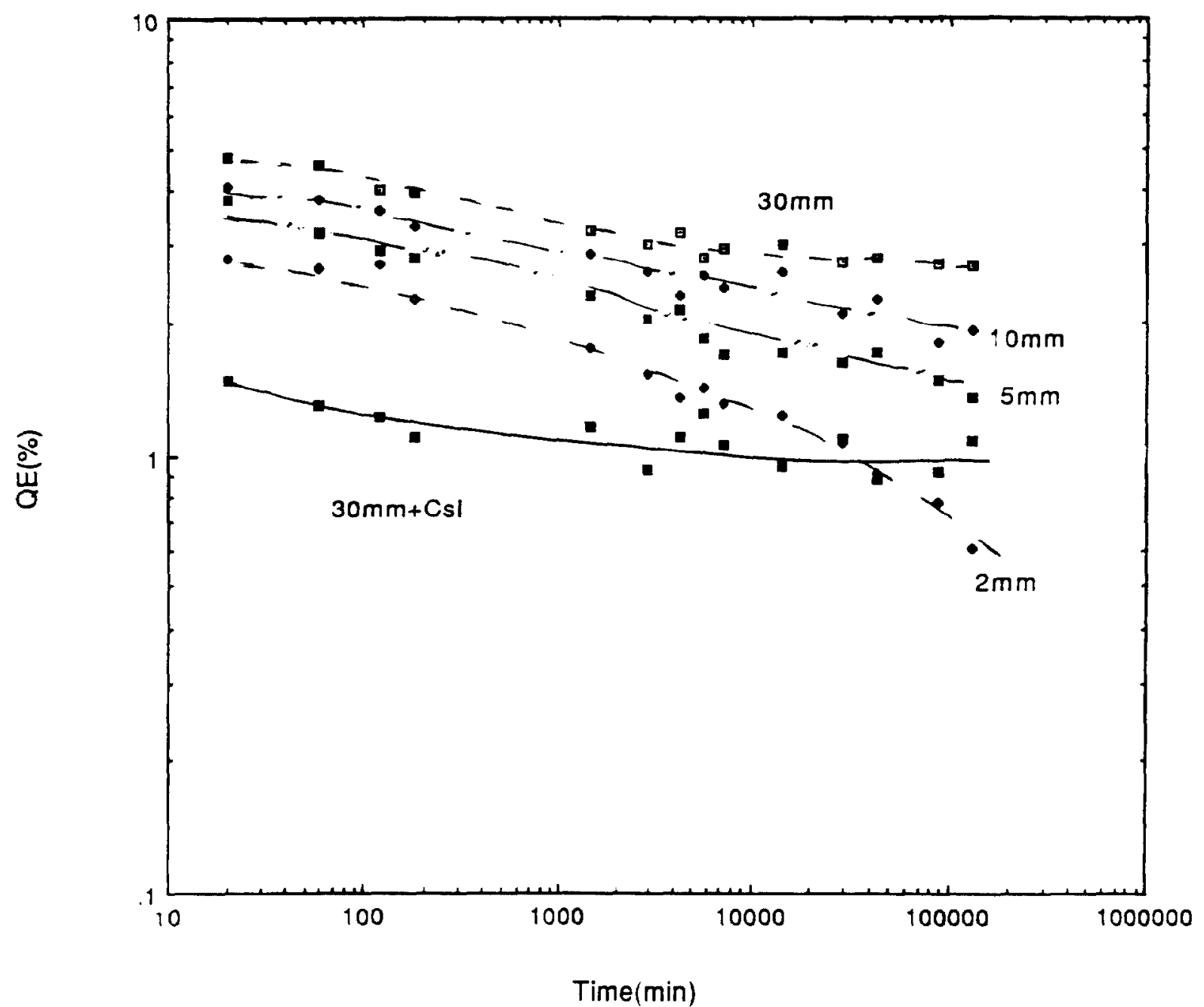

Fig. 3 


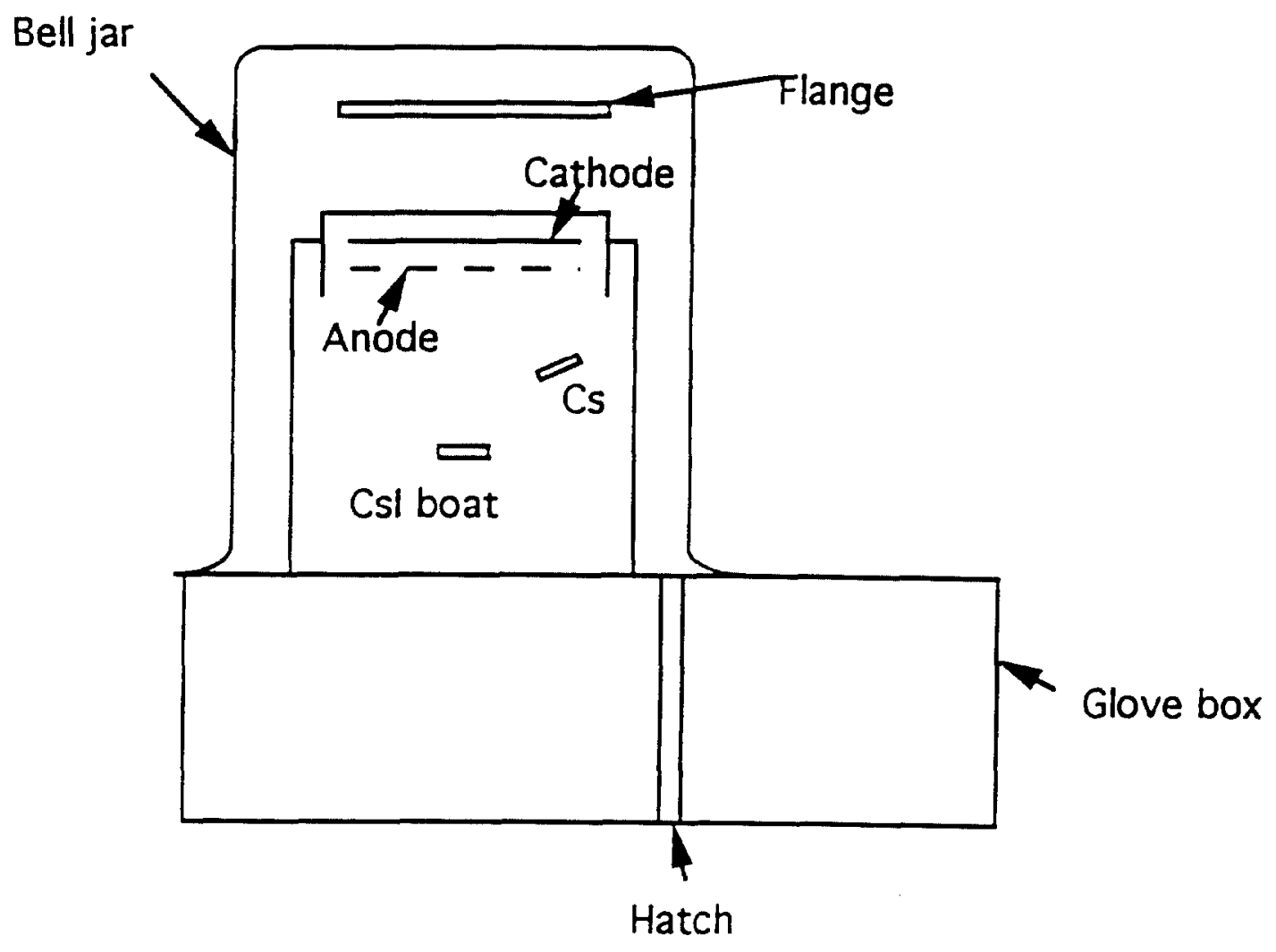

Fig.4 
Data from "Michigan GaAS"

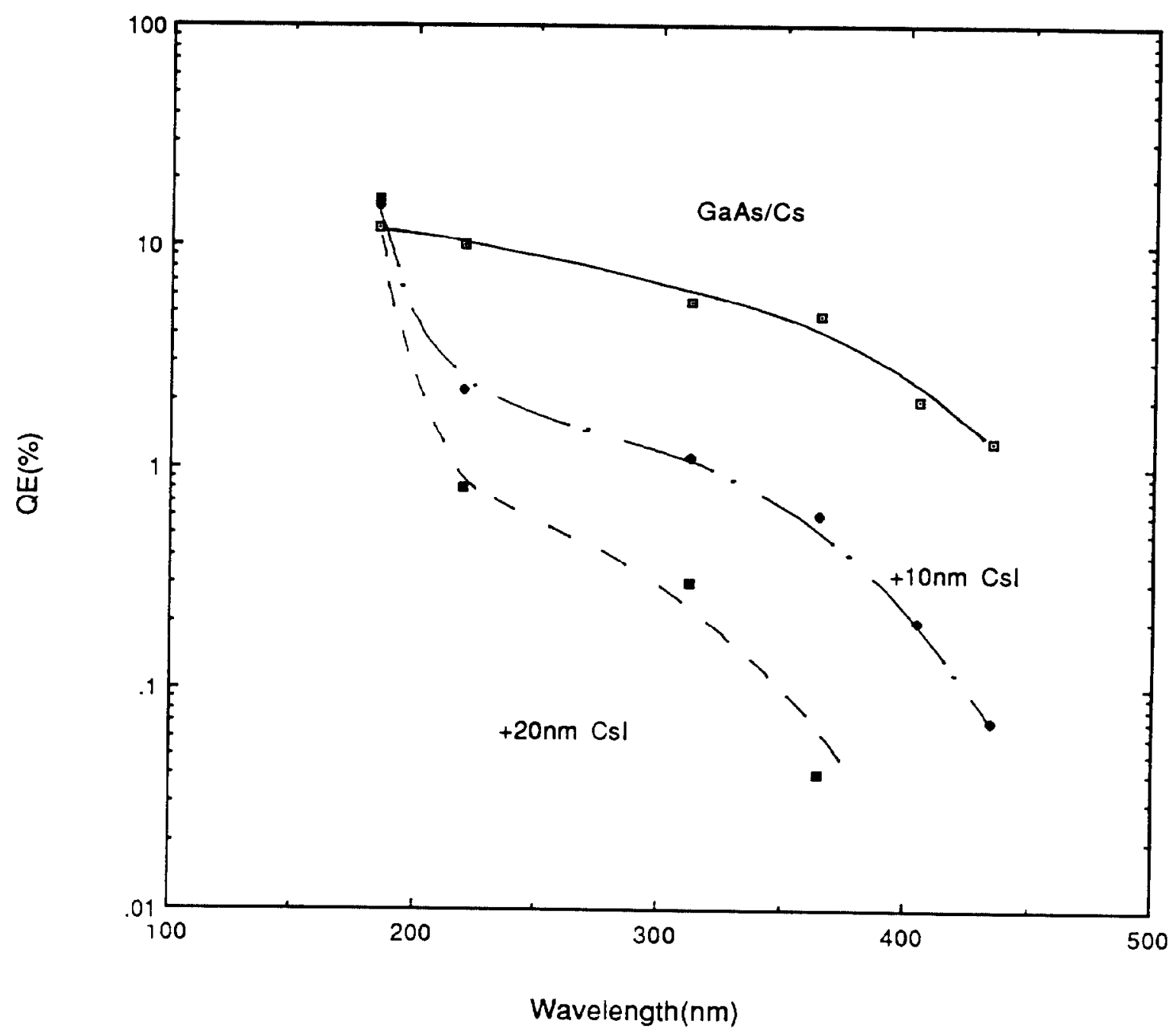

Fig. 5 


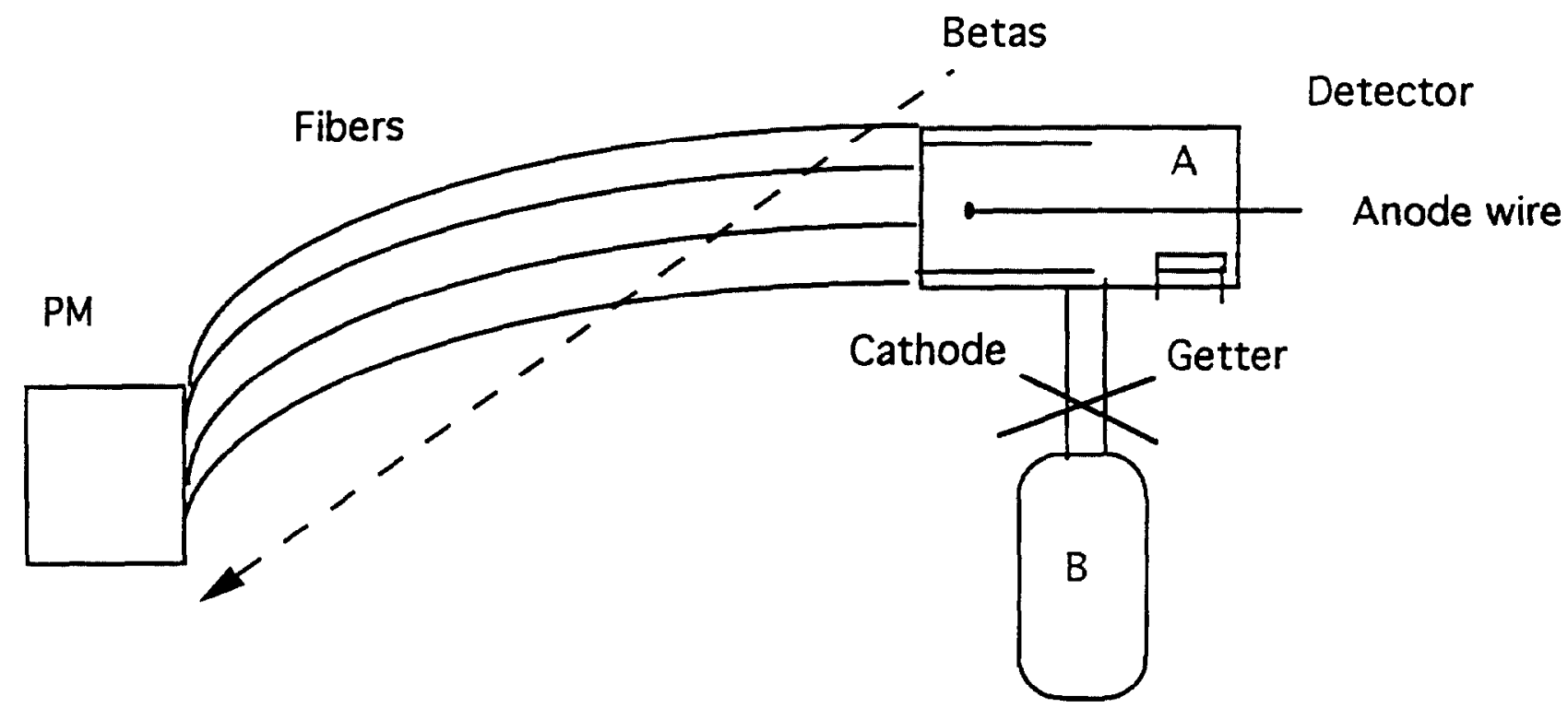

Fig. 6 
a)

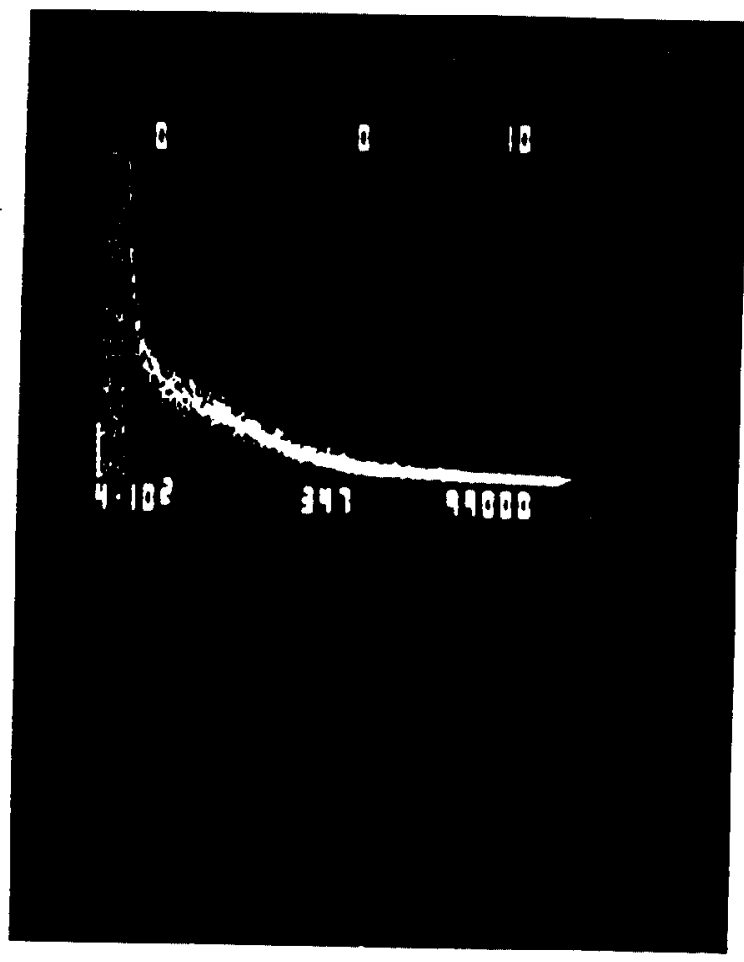

b)

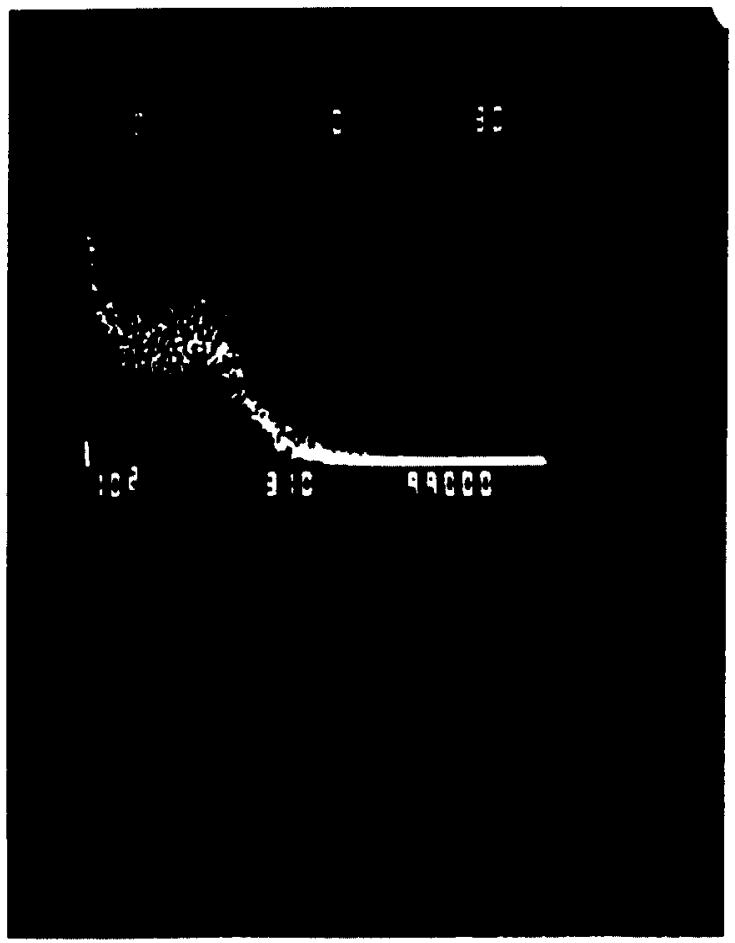

Fig. 7 


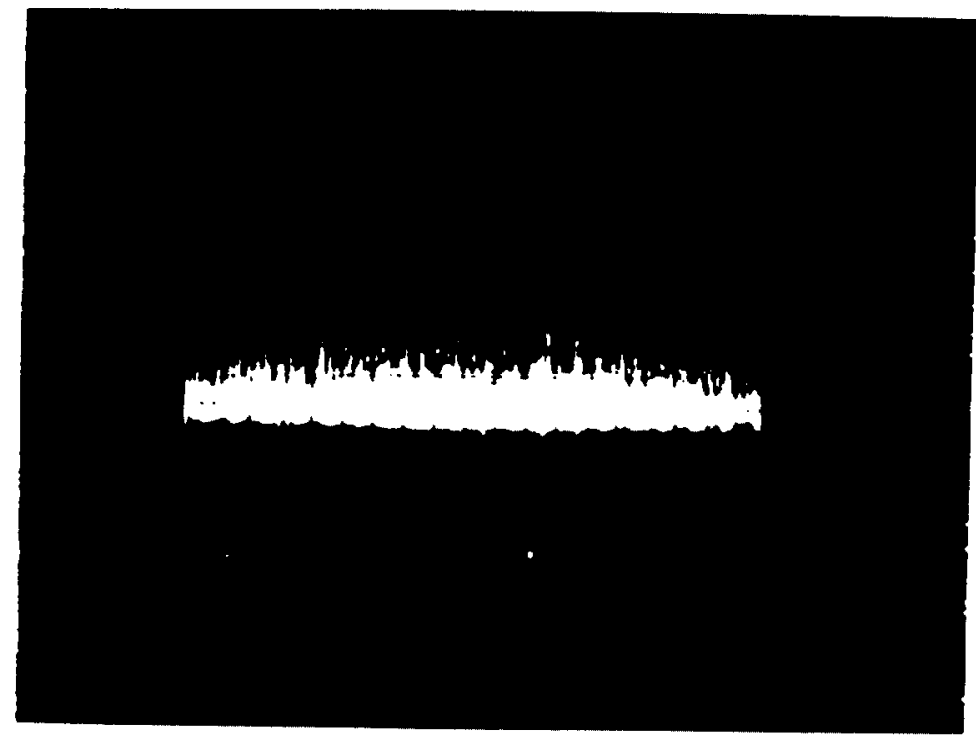

Fig. 8 